\title{
Fluorous methods for synthesis and separation of organic molecules*
}

\author{
Dennis P. Curran \\ Department of Chemistry, University of Pittsburgh, Pittsburgh, PA 15260 USA
}

\begin{abstract}
Fluorous molecules partition out of an organic phase and into a fluorous (highly fluorinated) phase in a liquid-liquid extraction. New fluorous techniques allow simple yet substantive separations of organic reaction mixtures based on the presence or absence of a fluorous tag. Fluorous-tagged molecules can also be separated from nontagged molecules by solid phase extraction over fluorous reverse-phase silica gel. This technique is ideal for solution-phase parallel synthesis because it allows simple yet substantive separations of organic reaction mixtures.
\end{abstract}

\section{STRATEGY LEVEL SEPARATIONS}

Research directed toward the discovery of small organic molecules with interesting functions is generally an exercise in organic synthesis. In this field, the conversion of an existing starting material into a new product occurs in a series of steps, each of which can usually be broken down into three stages (Fig. 1): 1) the reaction stage (the combining of substrates, reagents, solvents, etc. to make products); 2) the separations stage (the separation of the products from everything else in the reaction mixture); and 3) the identification and analysis stage. In this familiar process, the "reaction" stage of a synthetic step and the "separation" stage have traditionally been uncoupled. Reactions are executed, and then reaction mixtures are purified by using trial and error (or, more commonly, experience) to select a suitable purification technique.

In 1996 [1] and again in more detail in 1998 [2], we put forth a conceptual basis for uniting synthesis and purification based on familiar concepts of phase separation. In an extension of the concept of the "ideal synthesis", we introduced the concept of an "ideal purification" to be used until such time as "ideal syntheses" can be routinely achieved. In the "ideal purification", the desired products of the reaction mixture separate into a different phase from everything else that is present in the mixture. When this goal is met, reactions can be purified simply by workup. Workup includes the phase separation

1) Reaction Stage

Substrate $\frac{\begin{array}{l}\text { reactant(s) } \\ \text { reagent(s) }\end{array}}{\begin{array}{l}\text { catalytst(s) } \\ \text { solvent(s) }\end{array}}+\begin{gathered}\text { Product + byproducts } \\ \text { + recovered reaction components }\end{gathered}$

2) Purification Stage

a) workup

b) chromatography

\section{3) Identification/Analysis Stage:}

Product is characterized by NMR, IR, MS, X-ray, HPLC, etc.

Fig. 1 Stages of a step in organic synthesis.

\footnotetext{
*Lecture presented at the $13^{\text {th }}$ International Conference on Organic Synthesis (ICOS-13), Warsaw, Poland, 1-5 July 2000. Other presentations are published in this issue, pp. 1577-1797.
} 
techniques of extraction, evaporation, and filtration, and the phases that are readily separated by these common techniques are gases, aqueous liquids, organic liquids, and insoluble solids.

\section{THE FLUOROUS PHASE AND FLUOROUS BIPHASIC CATALYSIS}

The fluorous liquid phase consists of perfluorinated or very highly fluorinated organic liquids. We routinely use 3M's "FC" family of "fluorinert fluids" as fluorous solvents. For example, FC-72 is a solvent mixture consisting mostly of perfluorohexanes, and boils at about $56^{\circ} \mathrm{C}$. FC-72 and related solvents are immiscible both in water and in typical organic solvents, and effectively form a new phase for use in workup level separations.

The field of fluorous chemistry is quite young. Although not phrased in today's terminology, the 1991 thesis of Dr. M. Vogt in Aachen probably contains the first description of a fluorous reaction. This research was not published in the open literature until very recently, however [3]. Our research was precipitated by a short paper by Dong-Wei Zhu at 3M that described the use of FC-77 as a solvent for transesterification [4]. By distilling out the lower boiling alcohol into a Dean-Stark trap, Zhu drove an equilibrium reaction to completion. As far as we are aware, this was the first published example of an organic reaction conducted in a fluorous solvent.

In a seminal 1994 paper, Horváth and Rábai introduced the technique of fluorous biphasic catalysis (or FBC) [5]. Their original hydroformylation reaction is summarized in Fig. 2. A biphasic mixture of an alkene, toluene (the organic solvent), a fluorous phosphine, a standard rhodium salt, perfluoromethylcyclohexane (the fluorous solvent), $\mathrm{CO}$, and $\mathrm{H}_{2}$ was heated to effect reaction. Hydroformylation occurs, perhaps at the interface of the organic and fluorous phases. After cooling, the toluene phase containing the organic products is separated from the fluorous phase containing the catalyst. The fluorous phase can then be reused to catalyze another reaction, so the technique can effectively be considered as a liquid-phase catalyst immobilization method [6]. A number of these useful reactions employ organic/fluorous solvent pairings that are miscible at higher temperatures (thus allowing reactions to occur in a homogeneous medium) but that separate on cooling.

\section{FLUOROUS TECHNIQUES WITH FEWER FLUORINES}

Our work in the fluorous field diverges from fluorous biphasic catalysis in two important ways. First, we avoid fluorous reaction solvents. Fluorocarbons and related molecules are exceptionally poor solvents for organic molecules and are extremely nonpolar. Thus, the scope of reactions that succeed in either biphasic or homogenous solvent mixtures with large quantities of fluorous solvents is expected to be rather narrow. We prefer to use fluorous solvents at the separation stage, not the reaction stage. Second, we are now rapidly moving in the direction of using fluorous solid-phase extractions to replace liquid-liquid extractions for some applications.

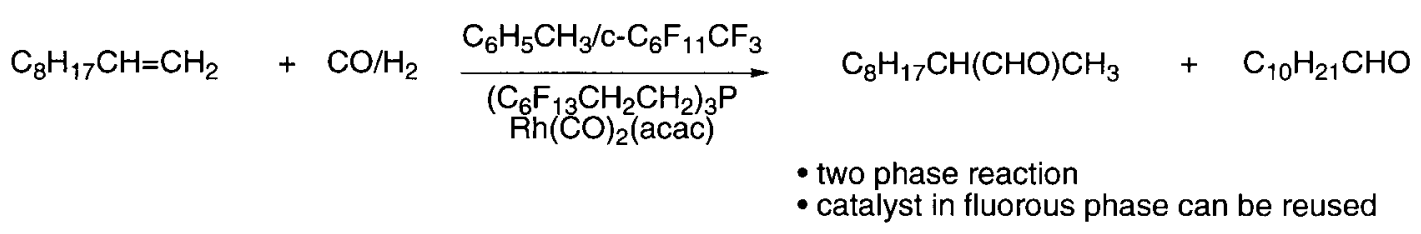

Fig. 2 The first example of "fluorous biphasic catalysis". 
The family of fluorous tin hydrides shown in Fig. 3 can be used in radical reactions as replacements for the traditional reagent, tributyltin hydride [7]. Reagents such as $\mathbf{1 a}$ and $\mathbf{1 c}$ have high partition coefficients into fluorous solvents, and can be readily removed by liquid-liquid extractions. However, fluorinated reaction solvents or cosolvents are needed to give such heavily fluorinated reagents solubility in reaction media [8]. The more lightly fluorinated reagents, as well as those with propylene spacers, have higher solubility in organic solvents. This result is a bonus at the reaction stage, where increased solubility is desirable, but a detraction at the separation stage since multiple liquid-liquid extractions are needed. However, a single-pass solid-liquid extraction over silica gel with a fluorocarbon-bonded phase [9] provides a simple and effective separation of the reaction mixture into organic and fluorous components.

In another set of fluorous techniques, substrates are "tagged" with fluorous groups for separation from untagged reagents, reactants, catalysts, etc. in an exercise that is conceptually analogous to solidphase synthesis [10]. Large numbers of fluorines can be used to impart solubility of the tagged substrates in fluorous solvents, but the high molecular weights of such compounds detract from the practicality of this approach.

Cutting back on the number of fluorines in the tag is expected to increase the organic solubility of tagged molecules (useful for reaction chemistry) while decreasing the fluorous solubility. The later effect poses an obvious problem when using liquid-liquid extractions, so light fluorous synthesis uses solid-liquid extractions instead. The left side of Fig. 4 shows the dramatic effect of fluorine substitution on retention times of a series of tagged amides 2a-i [11]. The control amide 2a (bearing an alkyl instead of a fluoroalkyl group) comes off with the solvent front, as do essentially all other nonfluorinated organic compounds under these conditions $(80 \% \mathrm{MeOH})$. As the eluting power of the solvent is increased, the amides bearing fluorinated tags come off in order of fluorine content. The separation is unique to fluorous silica and cannot be reproduced on normal phase or standard reverse phase supports. The separation must be expedited by a gradient; under isocratic conditions, the retention time of $\mathbf{2 h}$ is close to $1 \mathrm{~h}$, and $\mathbf{2 i}$ does not appear to come off at all (up to $2 \mathrm{~h}$ ). Most importantly, all tagged compounds 2 are soluble in dichloromethane and insoluble in FC-72. In other words, even 2i with 21 fluorines does not have nearly enough fluorines to be useful in liquid-liquid extractions (we estimate that about 50 fluorines would be needed).

Despite the lack of solubility of $\mathbf{2}$ in fluorocarbon solvents, huge retention differences between organic compounds (solvent front) and fluorous-tagged compounds ( $>30 \mathrm{~min}$ for the longer tags) trans-

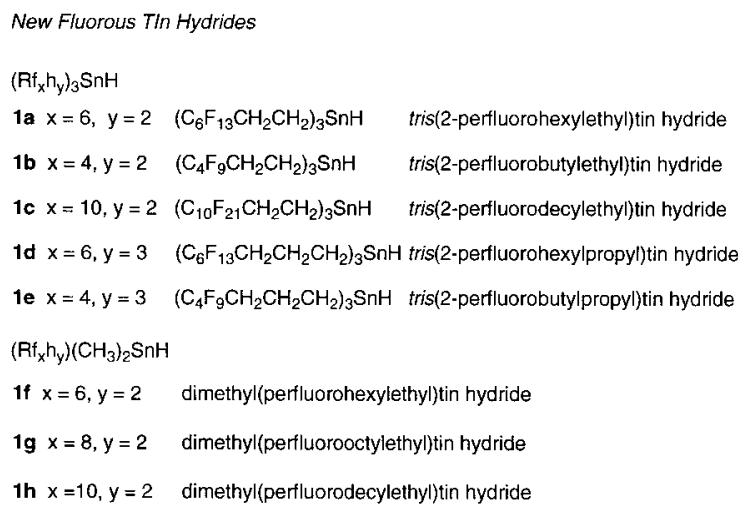

Fig. 3 A family of fluorous tin hydrides.
Partition Coefficients of Tin Hydrides

\begin{tabular}{c|l|c|c|c|c} 
& tin hydride & $\mathrm{F} / \mathrm{H}^{\mathrm{a}}$ & $\% \mathrm{~F}^{\mathrm{b}}$ & $\mathrm{FC}-72 / \mathrm{CH}_{3} \mathrm{CN}$ & $\mathrm{FC}-72 / \mathrm{C}_{6} \mathrm{H}_{6}$ \\
\hline & $\mathrm{Bu}_{3} \mathrm{SnH}$ & $\mathrm{NA}$ & 0 & $<1 / 150$ & $<1 / 350$ \\
1a & $\left(\mathrm{Ri}_{6} \mathrm{~h}_{2}\right)_{3} \mathrm{SnH}$ & 3.25 & 64 & $160 / 1$ & $45 / 1$ \\
1b & $\left(\mathrm{Rf}_{4} \mathrm{~h}_{2}\right)_{3} \mathrm{SnH}$ & 2.25 & 60 & $5.8 / 1$ & $10.5 / 1$ \\
1c & $\left(\mathrm{Rf}_{10} \mathrm{~h}_{2}\right)_{3} \mathrm{SnH}$ & 5.25 & 68 & high $^{\mathrm{c}}$ & high $^{\mathrm{c}}$ \\
1d & $\left(\mathrm{Rf}_{6} \mathrm{~h}_{3}\right)_{3} \mathrm{SnH}$ & 2.17 & 62 & $6.4 / 1$ & $10.0 / 1$ \\
1e & $\left(\mathrm{Rf}_{4} \mathrm{~h}_{3}\right)_{3} \mathrm{SnH}$ & 1.50 & 57 & $2.3 / 1$ & $1.2 / 1$ \\
1f & $\left(\mathrm{Rf}_{6} \mathrm{~h}_{2}\right) \mathrm{SnMe} \mathrm{Sn}_{2} \mathrm{H}$ & 0.87 & 50 & $2.4 / 1$ & $0.7 / 1$ \\
1g & $\left(\mathrm{Rf}_{8} \mathrm{~h}_{2}\right) \mathrm{SnMe}_{2} \mathrm{H}$ & 1.13 & 54 & $14 / 1$ & $2.5 / 1$ \\
1h & $\left(\mathrm{Rf}_{10} \mathrm{~h}_{2}\right) \mathrm{SnMe}_{2} \mathrm{H}$ & 1.40 & 57 & $48 / 1$ & $4.7 / 1$
\end{tabular}

a) ratio of fluorines to hydrogens; b) \% fluorine by molecular weight; c) due to low solubility, the partition coefficient measurements are not reliable. 
Separation of a Mixture of Amides 1a-i on a Fluofix 120E Column

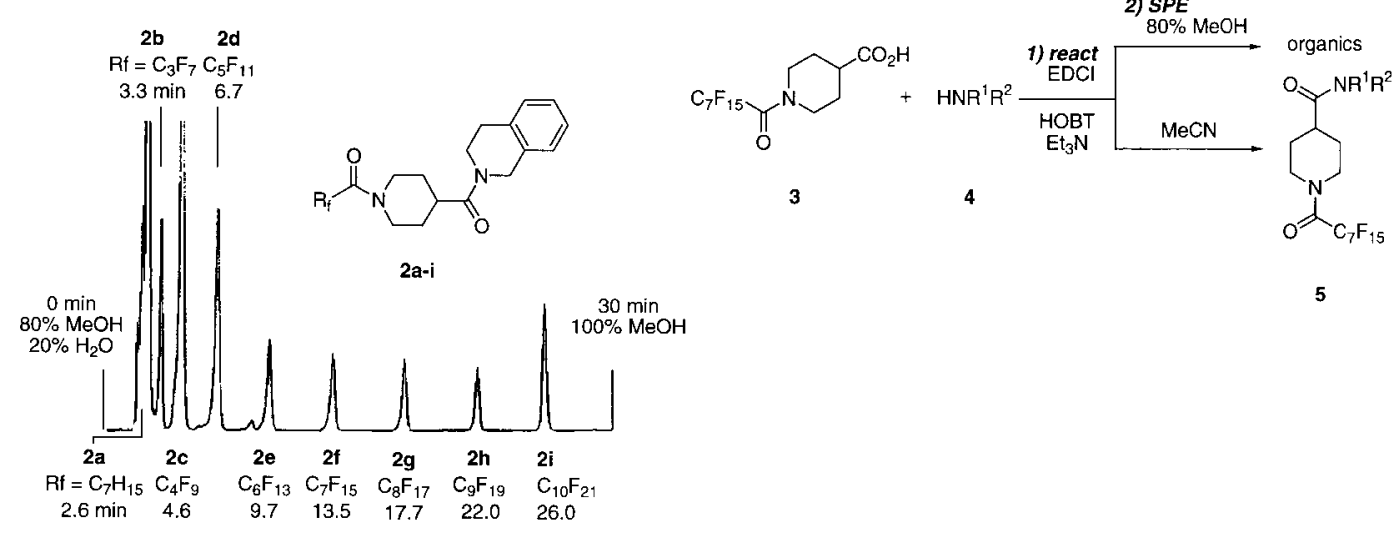

Fig. 4 A demonstration of light fluorous synthesis.

late into simple separations by solid-phase extractions, as shown in the demonstration experiment in the right side of Fig. 4.

A 16-compound library was made by the coupling of four fluorous-tagged amino acids with four amines. A representative example of this library is shown in Fig. 4. The acid $\mathbf{3}$ was coupled with amines 4 by using standard coupling conditions. The crude reaction mixture was eluted through fluorous silica first with $80 \% \mathrm{MeOH} / \mathrm{H}_{2} \mathrm{O}$ (to collect the organic fraction) and then with acetonitrile (to collect the fluorous fraction). Evaporation of the fluorous fraction provided pure products $\mathbf{5}$. Yields and purities were highly satisfactory for the library thanks to the efficient chemistry and the simple but substantive separation provided by the solid-phase extraction. Some problems were encountered with the cleavage of the fluorous acyl groups in this series of compounds, although several different reaction conditions were developed. More recently, we have developed fluorous analogs of the BOC group, which may be more generally useful for nitrogen protection than the fluorous acyl groups shown in Fig. 4.

\section{ACKNOWLEDGMENTS}

I warmly thank an enthusiastic group of coworkers for their intellectual and experimental contributions to this project. I also thank the National Science Foundation, the National Institutes of Health, WarnerLambert, CombiChem, and Merck for funding our work in this area.

\section{REFERENCES}

1. D. P. Curran. Chemtracts-Org. Chem. 9, 75-87 (1996).

2. D. P. Curran. Angew. Chem. Int. Ed. Engl. 37, 1175-1196 (1998).

3. W. Keim, M. Vogt, P. Wasserscheid, B. DriessenHolscher. J. Mol. Catal. A Chem. 139, 171-175 (1999).

4. D.-W. Zhu. Synthesis 953-54 (1993).

5. I. T. Horváth and J. Rábai. Science 266, 72-75 (1994).

6. I. T. Horváth. Acc. Chem. Res. 31, 641-650 (1998).

7. D. P. Curran, S. Hadida, S. Y. Kim, Z. Y. Luo. J. Am. Chem. Soc. 121, 6607 (1999); I. Ryu, T. Niguma, S. Minakata, M. Komatsu, Z. Y. Luo, D. P. Curran. Tetrahedron Lett. 40, 2367 (1999); I. Ryu, T. Niguma, S. Minakata, M. Komatsu, S. Hadida, D. P. Curran. Tetrahedron Lett. 38, 7883 (1997); D. P. Curran and S. Hadida. J. Am. Chem. Soc. 118, 2531 (1996). 
8. For use of microwave conditions, see: K. Olofsson, S. Y. Kim, M. Larhed, D. P. Curran, A. Hallberg, J. Org. Chem. 64, 4539 (1999).

9. D. P. Curran, S. Hadida, M. He. J. Org. Chem. 62, 6714 (1997).

10. A. Studer, S. Hadida, R. Ferritto, S. Y. Kim, P. Jeger, P. Wipf, D. P. Curran. Science 275, 823 (1997).

11. D. P. Curran and Z. Y. Luo. J. Am. Chem. Soc. 121, 9069 (1999). 